\title{
Assessing effects of pressure on tumor and normal tissue physiology using an automated self-calibrated, pressure- sensing probe for diffuse reflectance spectroscopy
}

Gregory M. Palmer

Hengtao Zhang

Chen-Ting Lee

Husam Mikati

Joseph A. Herbert

Marlee Krieger

Jesko von Windheim

Dave Koester

Daniel Stevenson

Daniel J. Rocke

Ramon Esclamado

Alaatin Erkanli

Nirmala Ramanujam

Mark W. Dewhirst

Walter T. Lee 


\title{
Assessing effects of pressure on tumor and normal tissue physiology using an automated self-calibrated, pressure-sensing probe for diffuse reflectance spectroscopy
}

\author{
Gregory M. Palmer, ${ }^{a, b, *}$ Hengtao Zhang, ${ }^{a}$ Chen-Ting Lee, ${ }^{a}$ Husam Mikati, ${ }^{a}$ Joseph A. Herbert, ${ }^{a}$ Marlee Krieger, \\ Jesko von Windheim, ${ }^{\mathrm{b}}$ Dave Koester, ${ }^{\mathrm{b}}$ Daniel Stevenson, ${ }^{\mathrm{b}}$ Daniel J. Rocke, ${ }^{\mathrm{d}}$ Ramon Esclamado, ${ }^{\mathrm{d}}$ \\ Alaatin Erkanli, ${ }^{\mathrm{e}}$ Nirmala Ramanujam, ${ }^{\mathrm{b}, \mathrm{c}}$ Mark W. Dewhirst, ${ }^{\mathrm{a}, \mathrm{c}}$ and Walter T. Lee ${ }^{\mathrm{d}, \mathrm{f}}$ \\ ${ }^{a}$ Duke University Medical Center, Department of Radiation Oncology, Durham, North Carolina, United States \\ ${ }^{b}$ Zenalux Biomedical, Inc., Durham, North Carolina, United States \\ 'Duke University, Department of Biomedical Engineering, Durham, North Carolina, United States \\ dDuke University Medical Center, Department of Surgery, Durham, North Carolina, United States \\ eDuke University Medical Center, Department of Biostatistics and Bioinformatics, Durham, North Carolina, United States \\ fDurham Veterans Affairs Medical Center, Durham, North Carolina, United States
}

\begin{abstract}
Diffuse reflectance spectroscopy (DRS) represents a quantitative, noninvasive, nondestructive means of assessing vascular oxygenation, vascularity, and structural properties. However, it is known that such measurements can be influenced by the effects of pressure, which is a major concern for reproducible and operator-independent assessment of tissues. Second, regular calibration is a necessary component of quantitative DRS to account for factors such as lamp decay and fiber bending. Without a means of reliably controlling for these factors, the accuracy of any such assessments will be reduced, and potentially biased. To address these issues, a self-calibrating, pressure-controlled DRS system is described and applied to both a patient-derived xenograft glioma model, as well as a set of healthy volunteers for assessments of oral mucosal tissues. It was shown that pressure had a significant effect on the derived optical parameters, and that the effects on the optical parameters were magnified with increasing time and pressure levels. These findings indicate that not only is it critical to integrate a pressure sensor into a DRS device, but that it is also important to do so in an automated way to trigger a measurement as soon as possible after probe contact is made to minimize the perturbation to the tissue site. @ 2018 Society of Photo-Optical Instrumentation Engineers (SPIE) [DOI: 10.1117/1.JBO.23.5.057004]
\end{abstract}

Keywords: diffuse reflectance spectroscopy; pressure; tissue; spectroscopy.

Paper 170775R received Nov. 28, 2017; accepted for publication Apr. 25, 2018; published online May 15, 2018.

\section{Introduction}

Tumor nodes metastasis (TNM) staging provides prognostic information and guides therapeutic decision-making in newly diagnosed patients with head and neck squamous cell carcinoma (HNSCC) and other cancers. However, the anatomically based TNM system has significant deficiencies. Patients presenting with the same stage disease can exhibit markedly different outcomes despite receiving identical treatments. Investigators have, therefore, sought to identify additional physiological and biological prognostic/predictive factors to augment TNM-derived data. Technologies that allow for in situ characterization of the underlying biology of both tumors and normal organs will provide a unique opportunity to address this problem. When obtained prior to the initiation of therapy this information can potentially improve the identification of those patients who are at high risk for failure with standard therapies and who might benefit from therapeutic intensification regiment. The ability to identify an unfavorable response very early (the first or second week) in the course of any given treatment would present more

*Address all correspondence to: Gregory M. Palmer, E-mail: greg.palmer@ duke.edu options for making meaningful changes in strategy than would be available closer to the end of therapy.

We have previously demonstrated that quantitative diffuse reflectance spectroscopy (DRS) can be used to assess diagnostically and therapeutically relevant endpoints in HNSCC, breast, cervix, and other cancers. However, prior studies have been hampered by the lack of a rigorous system for real-time assessment of probe pressure, and system throughput calibration, both of which we have shown to significantly affect accuracy and reproducibility. ${ }^{1,2}$

Calibration is required to compensate for the wavelength-dependent instrument response, lamp intensity fluctuations, and fiber bending losses. ${ }^{3,4}$ Current calibration techniques typically rely on measurements using standards and/or tissue phantoms, typically after the clinical measurements are completed. There are a number of limitations associated with such calibration methods. First, because the calibration is performed at the beginning or end of the study, real-time instrument fluctuations, such as lamp drift and fiber bending loss, cannot be compensated by these approaches. Second, they can require an additional $30 \mathrm{~min}$ 
for lamp warm-up and another 10 to 20 min for calibration, which is a significant amount of time in a clinical setting.

Uncontrolled probe-to-tissue coupling and pressure can make it difficult to obtain a reproducible spectrum, particularly for an untrained operator. Chan et al. $^{5}$ found that there was a decrease in the diffuse reflectance and an increase in the scattering coefficient between 400 and $1800 \mathrm{~nm}$ with compression of in vitro human skin. Reif et al. ${ }^{6}$ reported a study in which reflectance measurements were obtained in vivo from mouse thigh muscles while varying the contact pressure of the fiber-optic probe. They found that the blood vessel radius, oxygenation, and Mie theory slope decreased with pressure, while the reduced scattering coefficient at $700 \mathrm{~nm}$ increased as a function of pressure. Recently, Ti and Lin $^{7}$ studied the short- and long-term effects of probe pressure on in vivo diffuse reflectance using an animal model. They concluded that elevation in probe pressure induces major alterations in the profile of the reflectance spectra between 400 and $650 \mathrm{~nm}$ and the changes in the tissue optical properties depend on probe pressure, and tissue type.

We previously conducted serial baseline-corrected oxygen saturation $\left(\mathrm{SO}_{2}\right)$ derived from DRS measurements from a murine model of human head and neck cancer $(\mathrm{FaDu})$ measured in the first two weeks after a single dose of radiation. Using these data, we were able to discriminate between complete responders (no evidence of tumor over a 120-day period) and partial responders (tumor persistence within a 120-day period) before differences in tumor volume could be observed. ${ }^{8}$ No contrast was observed in total hemoglobin concentration [THb] or the average reduced scattering coefficient $\left(\mu_{s}^{\prime}\right)$. We have also shown that DRS can be used to extract tissue contrast in [Thb], $\mathrm{SO}_{2}$, and $\mu_{s}^{\prime}$ in HNSCCs in clinical studies. ${ }^{8,9} \mathrm{Hu}$ et al. ${ }^{9}$ demonstrated that a logistic regression model incorporating measurements of $\mathrm{SO}_{2}, \log [\mathrm{THb}]$, and $\log \left(\mu_{s}^{\prime}\right)$ resulted in a receiver operator curve with an area under the curve of 0.84 for all tissue types (from 112 normal and 36 SCC samples). Further, we have shown that pressure control ${ }^{2}$ and self-calibration ${ }^{10}$ can significantly reduce systematic error in the measurement of [THb] and $\mu_{s}^{\prime}$, respectively. Given the promising results of DRS applied to diagnostic and therapeutic applications in HNSCC, it is anticipated that these capabilities will be substantially improved through the incorporation of self-calibration and pressure-sensing capabilities.

\section{Methods and Results}

The Zenalux PC2 (Durham, North Carolina) diffuse optical spectroscopy system was used for all measurements. The optical components consist of a visible light spectrometer and lamp (Ocean Optics USB2000 and HAL2000) along with a custom fiber optic probe. This consisted of a pair of 486-micron core plastic optical fibers for the animal study, and a 750-micron core diameter plastic optical fiber for the clinical study, each with $0.51 \mathrm{NA}$. The large core size in the plastic fiber used clinically was necessary given its greater losses relative to glass, and to minimize the required exposure time for clinical use. For each fiber optic probe assembly, an edge-to-edge source-detection fiber separation distance of $0.15 \mathrm{~mm}$ was used at the tissue interface. A 2-m furcation tube assembly carries the fibers back to SMA 905 connectors that attach to the spectrometer and lamp.

\subsection{Pressure Sensing}

To enable pressure sensing while minimizing the effects of cable tension, there is a section of bare fiber between the furcation and ferule tubes. This bare fiber section is coiled inside of the probe handle, see Fig. 1. The furcation tube is rigidly attached to the handle and the ferule can move independently from it. The fiber coil shrinks or expands to take up the difference between the ferule and furcation tube separation distance over a nominal $1-\mathrm{cm}$ range of motion. The fibers were selected based on their ability to wind around the relatively tight fiber coil $(4-\mathrm{cm}$ diameter).

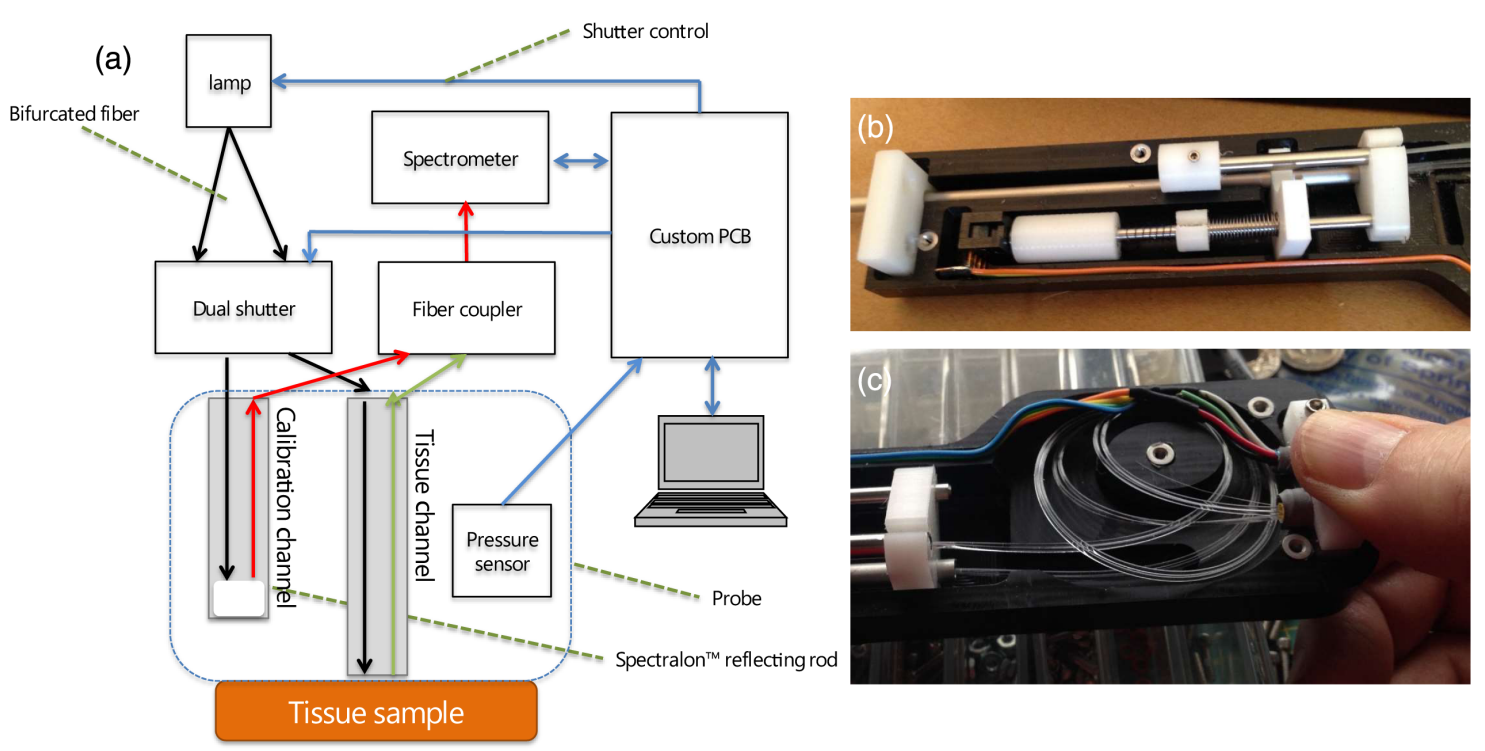

Fig. 1 An instrument schematic shows the system components. (a) The features include an optical switch (dual shutter) to direct light to either a calibration or tissue-sensing channel for automated calibration measurement. (b) A pressure sensing system was also integrated into the probe handle using a spring-loaded metal ferrule that contacted a force sensor. (c) This was uncoupled from the weight of the cable through use of a coiled segment of bare cable that could flex as the fiber tip was compressed against the sample. 


\subsection{Self-Calibration}

A system block diagram is shown in Fig. 1(a). The primary innovation is the incorporation of two optical paths: a calibration leg and a measurement leg to facilitate self-calibration with an automatic shutter. The calibration leg is comprised of a short (internally contained) ferule that is terminated with a plastic cap containing a spacer and a Spectralon ${ }^{\mathrm{TM}}$ calibration disk. In this way, calibration measurements can be obtained immediately after an experimental measurement with the probe in the same position and nearly the same time, which minimizes the effects of (1) lamp warm up or power drift and (2) effects of fiber bending. Past designs for self-calibration have used two spectrometers: one spectrometer for tissue measurements and another one for calibration. ${ }^{1,2}$ However, for both cost and performance reasons this is not the ideal configuration; it is unlikely that any two spectrometers will operate the same, throwing the SC measurements into doubt. Our updated design (Fig. 1) addresses this challenge using only one spectrometer with a $1 \times 2$ optical switch to rapidly switch back and forth between tissue measurements and calibration measurements.

\subsection{Analysis}

Data were background subtracted by taking a measurement with the lamp shutter closed. Data were also normalized to the calibration standard measurement to obtain the diffuse reflectance relative to the spectrally flat standard. A scalable inverse Monte Carlo-based algorithm was used to extract the underlying tissue optical properties. ${ }^{11}$ The model utilized measured data from 500 to $600 \mathrm{~nm}$, in $2.5-\mathrm{nm}$ increments to obtain a least squares fit. The model assumed absorption could be characterized by a combination of oxy and deoxy hemoglobin, modified by the pigment packing factor of van Veen et al., ${ }^{12}$ and a nonmelanin skin absorption component. ${ }^{13}$ Scattering is approximated by the relationship, $\mu_{s}^{\prime}=A \times \lambda^{-b}$, where $\mu_{s}^{\prime}$ is the reduced scattering coefficient, $A$ is the scattering amplitude, $\lambda$ is the wavelength in nanometers, and $b$ is the scatter power factor, respectively. The model inputs including absorber concentrations and scattering parameters were adjusted to obtain a least squares fit to the measured data using the Python SciPy library. ${ }^{14}$

A linear mixed effects model was used to test for significant main effects for pressure (modeled as a factor), time (represented by repeated sample number and modeled as a continuous variable), and site (modeled as a factor for the clinical measurements). Both hemoglobin oxygen saturation and total hemoglobin content were used as response variables to test for effects of these parameters on each. The nlme library in $R$ was used for the analysis using the lme function. ${ }^{15}$ The subject was modeled as a random effect, with pressure, time, and site input as fixed effects.

\subsection{Patient-Derived Xenograft Tumor Study}

Animal studies were performed in accordance with a protocol approved by the Duke Institutional Animal Care and Use Committee (IACUC). About 6-week-old female athymic nude mice were obtained from Duke breeding core facility. Human glioma patient-derived xenograft (PDX) tumors (kindly provided by Dr. Stephen Keir, Duke University) were implanted subcutaneously in the flank of the nude mice. Six nude mice were transplanted with glioma PDX tumors in the flank. When tumors were $\sim 300 \mathrm{~mm}^{3}$, the Zenascope was used to acquire spectra at a series of different pressures as assessed by the force sensor to produce calibrated pressure measurements (24, $48,72,120,192 \mathrm{mmHg}$ ). This was done by the technician, who placed the probe and attempted to match the pressure as closely as possible to the target value prior to collecting the measurement $( \pm 20 \%)$. The median exposure time was $1570 \mathrm{~ms}$. The PDX model was chosen as this has been demonstrated to more faithfully replicate the structural and functional characteristics of human tumors, as compared to cell line xenograft
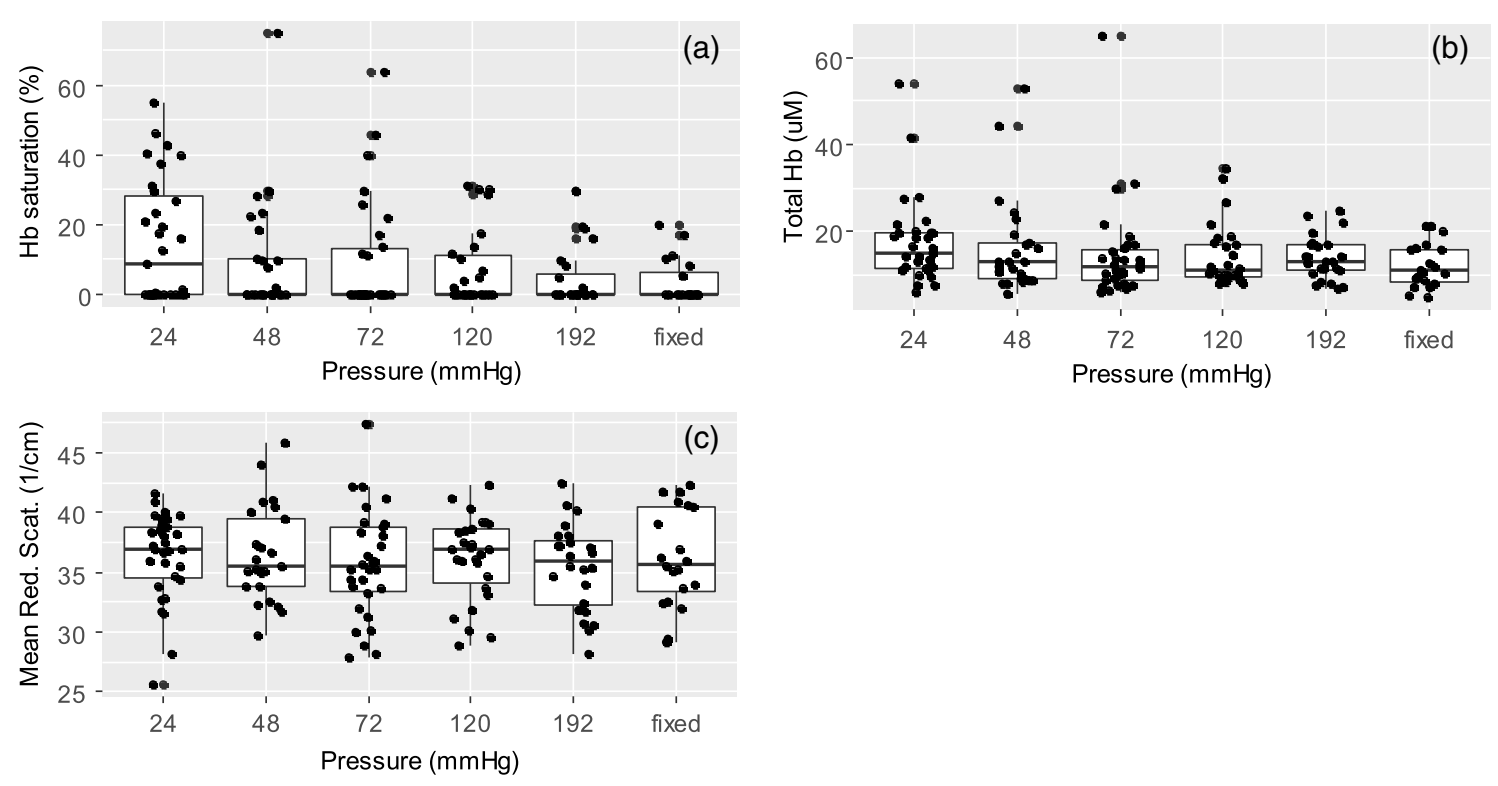

c)

Fig. 2 The optical outputs as a function of the nominal applied pressure. Each point corresponds to a single measurement ( $n=6$ animals with five repeated measurements each). It can be seen that the (a) $\mathrm{Hb}$ saturation exhibits a systematic decrease with increasing applied pressure. (b) The total $\mathrm{Hb}$ and (c) mean reduced scattering coefficient can be seen to be relatively more consistent across pressure. The case of a fixed probe is also shown, which appears similar to the higher pressure levels. 
models. ${ }^{16}$ After each measurement, the probe was removed from the tissue, and replaced for five repeated measurements at each pressure level. In addition, to replicate the typical sensing method, the spring-mounted probe was fixed in place, and the probe was placed similarly, which the technician (who was not experienced in optical spectroscopy techniques and blinded to the study intent) was instructed to place the probe using minimal pressure while ensuring complete contact with the tissue. This is referred to as the "fixed" probe measurement.

Figure 2 shows box and scatter plots of the full dataset. It can be seen that the hemoglobin oxygen saturation (HbSat) is significantly affected by pressure, with higher pressures resulting in lower HbSat levels ( $p=0.02$ by linear mixed effects model). Other parameters (total hemoglobin concentration and mean reduced scattering coefficient) did not show a significant difference. The fixed probe measurement appears similar to the higher pressure level for all parameters.

Figure 3 shows a similar plot looking at the intra-animal change in these parameters, focusing on those related to absorption as scattering is not significantly affected. The mean endpoint value obtained at lowest pressure measurement (24 mmHg) was taken as the baseline for each animal, and was subtracted from the averaged endpoint obtained for each subsequent pressure level. It can be seen that in general there is a suppression in all of the parameters as a function of pressure, but that $\mathrm{HbSat}$ and oxygenated $\mathrm{Hb}$ concentration change more consistently in a negative direction (all subjects appear at or below zero for the highest pressure level as well as the fixed probe case). This may explain why HbSat showed a significant effect of pressure, whereas total hemoglobin concentration did not (Table 1).
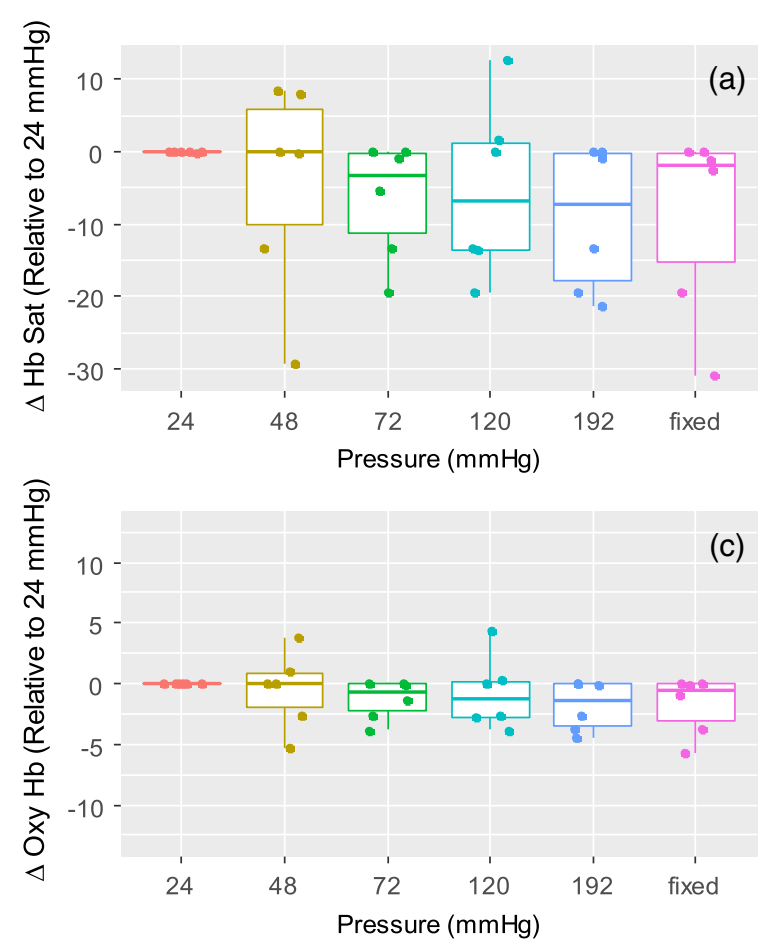

Table 1 Statistical significance for repetition number and pressure level using a linear mixed effects model for both hemoglobin saturation and total hemoglobin content for PDX tumor model.

\begin{tabular}{lcc} 
Parameter & $\begin{array}{c}\text { Hb saturation } \\
p \text {-value }\end{array}$ & $\begin{array}{c}\text { Total } \mathrm{Hb} \\
p \text {-value }\end{array}$ \\
\hline Repetition number (continuous) & 0.11 & 0.96 \\
Pressure level (factor) & $0.02^{1 \star}$ & 0.46 \\
\hline
\end{tabular}

* $p<0.05$

\subsection{Volunteer Study}

To study the influence of pressure in a realistic clinical setting, a healthy volunteer study was initiated at Duke University Medical Center, which was approved by the Duke University Institutional Review Board. To minimize the time required for measurement and simulate a more realistic clinical measurement mode, the software was updated to enable automated pressure sensing and repeated measurements using a single probe placement. This was achieved through continuous sampling of the force sensor upon activation of the probe along with continuous sampling of the spectrometer for the optical measurement. For each spectra obtained, it was checked if the force sensor (calibrated to units of pressure) remained within the specified valid range during the entire measurement, as well as the sensor readings immediately before and after the spectra were obtained. Three pressure levels were tested. This process was repeated 10 times per measurement site and pressure
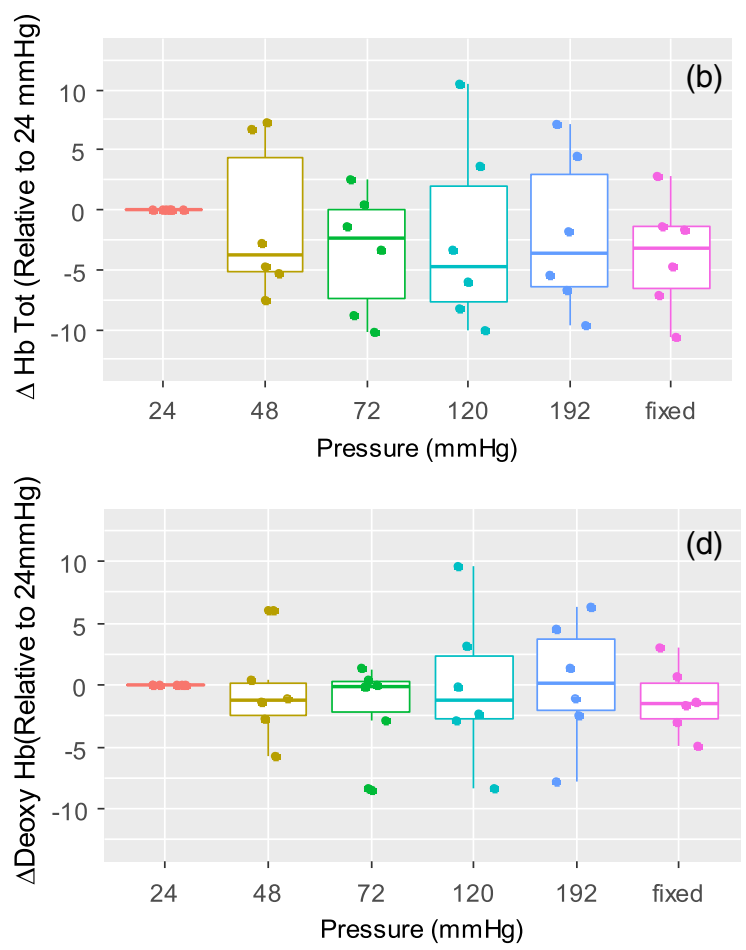

Fig. 3 The relative in change for each optical endpoint is shown for each animal. The average of five repeated measurements was taken for each animal for each pressure level, and the lowest pressure level measurement was subtracted from this to obtain the change in signal. The most consistent changes are seen for (a) $\mathrm{Hb}$ saturation and (c) oxy $\mathrm{Hb}$ concentration, both shows a median decrease with increasing pressure. (b) The total hemoglobin, and (d) deoxy hemoglobin also show decreasing trends with increasing pressure. 
setting. Four sites were measured in each subject, cheek, tongue, sublingual, and gums. Each measurement set (site and pressure combination) took a median time of three seconds to acquire 10 replicates plus one calibration measurement. The median exposure time per acquisition was $182 \mathrm{~ms}$.

Figure 4 shows boxplots of each endpoint, broken down by site and pressure levels. It can be seen that for any given site, there is a consistent decrease in the median hemoglobin saturation as a function of applied pressure. In contrast, the total hemoglobin concentration decreases in some cases but not for all. The linear mixed effects model indicates however, that for both $\mathrm{Hb}$ saturation, as well as Total $\mathrm{Hb}$, the site, and applied pressure are independently significant factors, whereas measurement repeat number is also significant for $\mathrm{Hb}$ saturation (Table 2). When broken down into oxy and deoxy Hb concentrations, it can be seen that the oxy $\mathrm{Hb}$ shows a consistent decrease, whereas deoxy $\mathrm{Hb}$ does not, and in some cases even increases with increasing pressure. Thus the consistent change in $\mathrm{Hb}$ Saturation appears to be driven by a consistent drop in the concentration of oxy $\mathrm{Hb}$. Finally, mean reduced scattering does not show any observable trend with pressure applied [Fig. 4(e)].

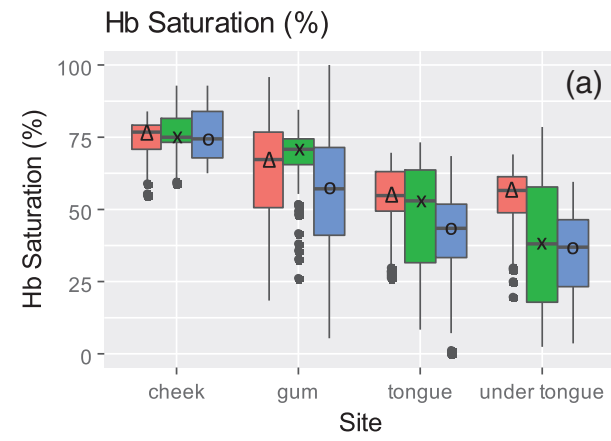

a)

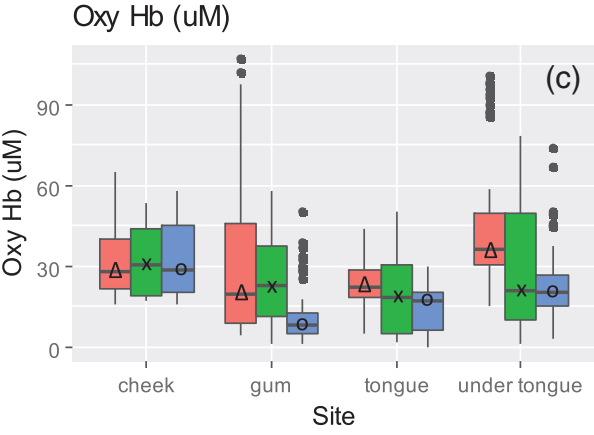

(c)

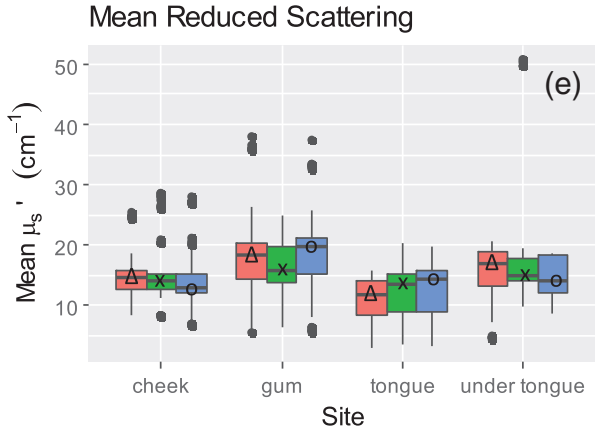

(e)

$$
\begin{aligned}
& \text { Pressure } \\
& \text { 㐫24-72 } \\
& \text { 这72-120 } \\
& \text { 田120-168 }
\end{aligned}
$$

In further examining the intra-subject change in these parameters as a function of pressure, the lowest pressure measurements were averaged and subtracted from the averaged signal from each of the other pressure levels for each subject (Fig. 5). In this case, it can be seen that there are consistent drops in the median hemoglobin saturation, total $\mathrm{Hb}$, and oxy $\mathrm{Hb}$ at the higher pressure levels, which appear, in general, to increase in magnitude with increasing pressure.

Table 2 Statistical significance for the monitored parameters using a linear mixed effects model for both hemoglobin saturation and total hemoglobin content for the volunteer study.

\begin{tabular}{lcc}
\hline Parameter & $\begin{array}{c}\text { Hb saturation } \\
p \text {-value }\end{array}$ & $\begin{array}{c}\text { Total Hb } \\
p \text {-value }\end{array}$ \\
\hline Repetition number (continuous) & $<0.0001^{1 *}$ & 0.23 \\
Pressure level (factor) & $<0.0001^{*}$ & $<0.001^{*}$ \\
Site (factor) & $<0.0001^{*}$ & $<0.001^{*}$ \\
\hline
\end{tabular}

${ }^{*} p<0.05$

Total $\mathrm{Hb}(\mathrm{uM})$

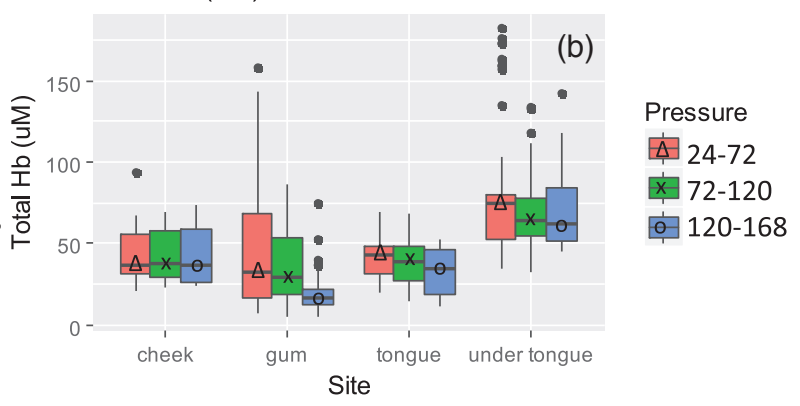

Deoxy $\mathrm{Hb}(\mathrm{uM})$

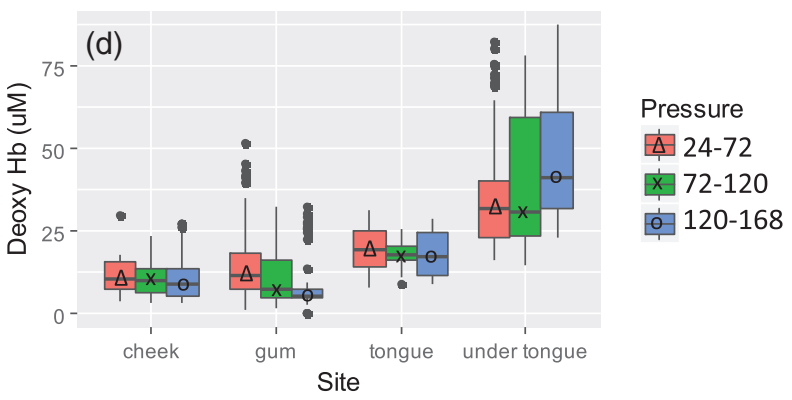

Fig. 4 Optical parameters from a set of nine healthy volunteers. A series of 10 measurements were acquired sequentially from each of the four sites from each subject, for each of the three target pressure ranges. Deviations as a function of pressure can be seen in particular for (a) $\mathrm{Hb}$ saturation, and (c) oxy $\mathrm{Hb}$ concentration, with other parameters, namely (b) total $\mathrm{Hb}$, (d) deoxy $\mathrm{Hb}$, and (e) mean reduced scattering coefficient, showing more variable responses. 

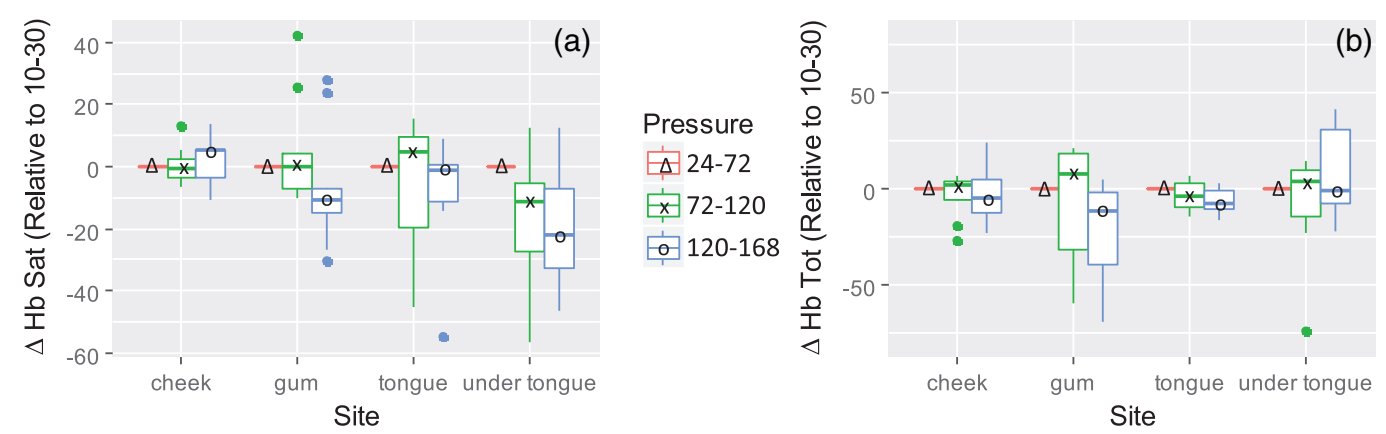

(b)
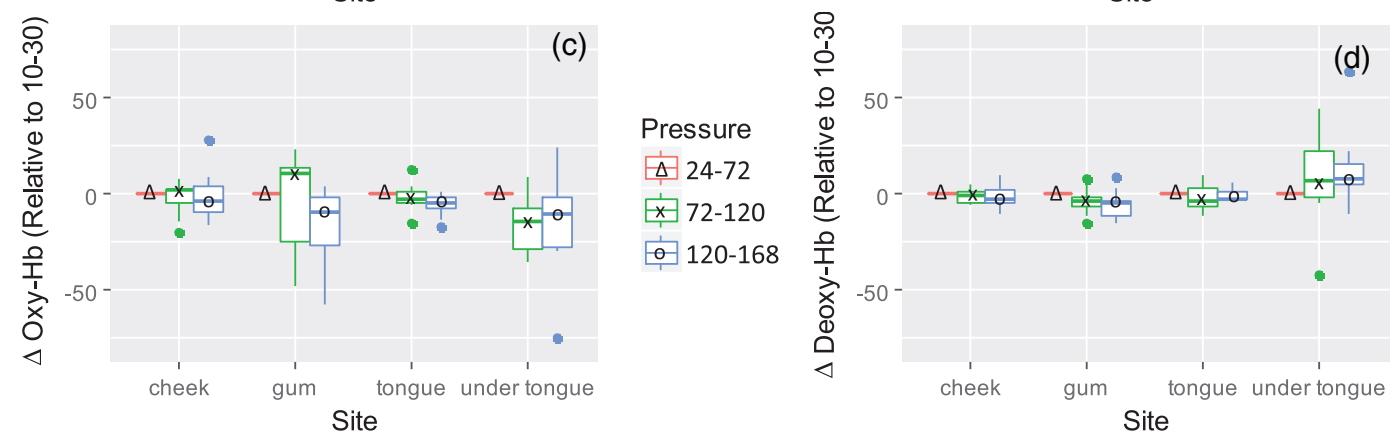

Pressure

$\triangle$ 24-72

安 72-120

- 120-168

Pressure

$\triangle 24-72$

$x$ 72-120

120-168

Fig. 5 Relative change in optical parameters relative to the lowest pressure is shown with respect to tissue site and pressure level. It can be seen that the largest and most consistent deviations appear for (a) $\mathrm{Hb}$ saturation and (c) oxy-Hb concentration, with more consistent values seen for (b) total hemoglobin, and (d) deoxy $\mathrm{Hb}$.

(a)

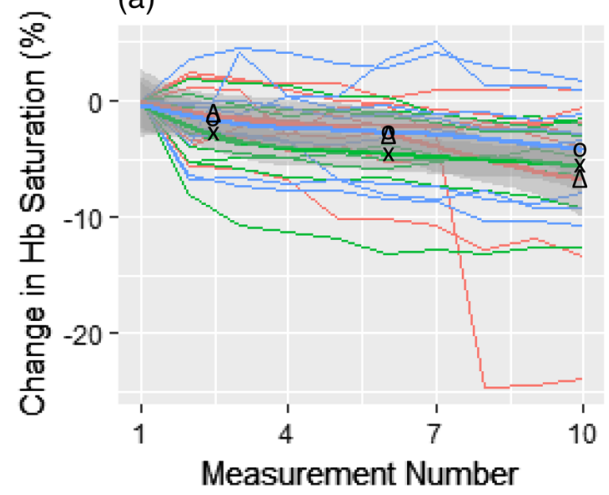

(c)

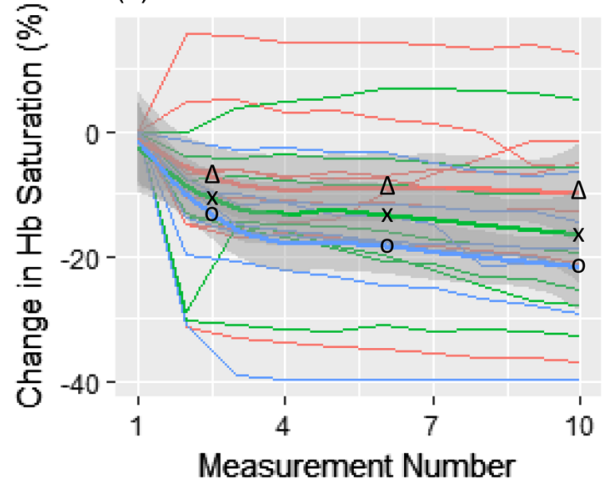

(b)
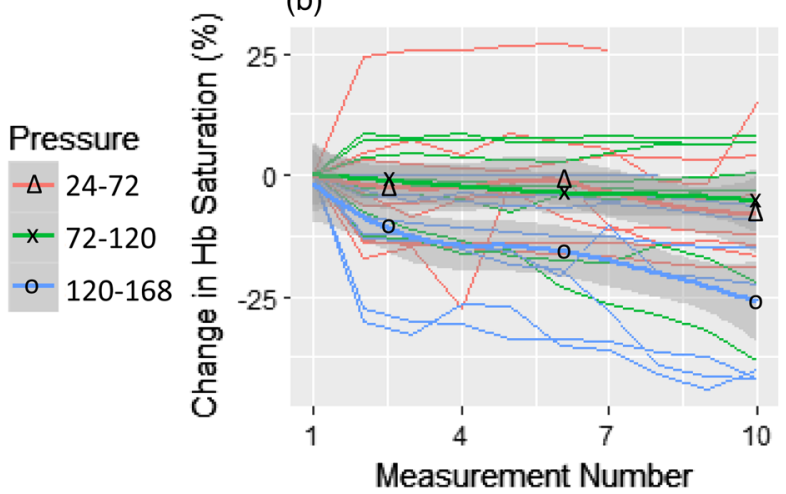

(d)

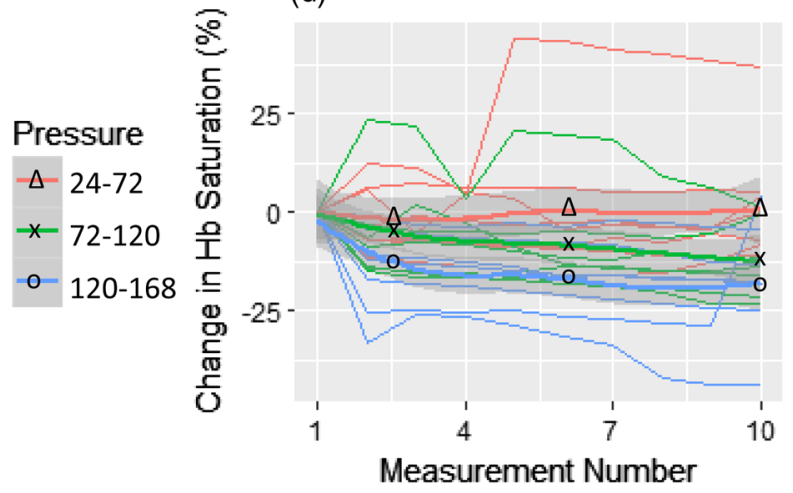

Pressure

$\triangle$ 24-72

72-120

$120-168$
Pressure

$\triangle$ 24-72

72-120

120-168

Fig. 6 For volunteer subject measurements, the $\mathrm{Hb}$ saturation measurement as a function of repeated measurement number is shown for each of the nine subjects at each of the three pressure levels. The moving average mean (loess) is also shown along with the $95 \%$ confidence intervals. This is shown for (a) cheek, (b) gum, (c) tongue, and (d) under the tongue. It can be seen that the mean $\mathrm{Hb}$ saturation significantly drops for the majority of the site-pressure combinations with increasing reduction over time. In addition, the higher pressure levels in general can be seen to have a larger drop over time. 
(a)

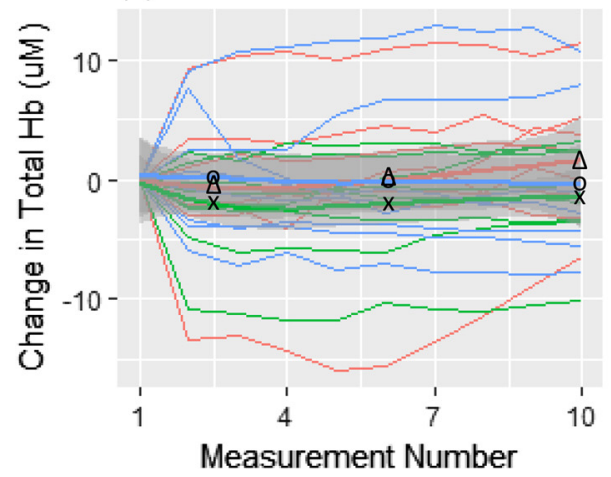

(c)

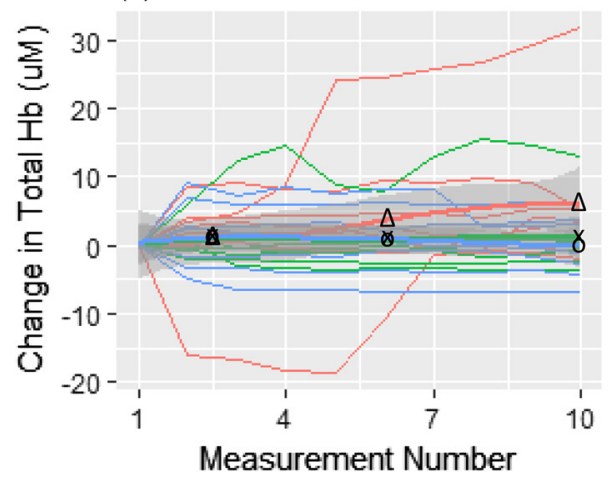

(b)
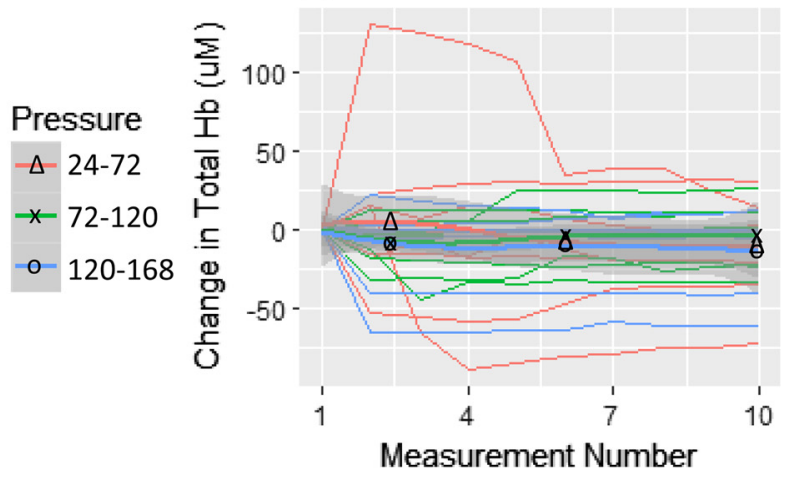

Pressure

$\triangle 24-72$

$72-120$

120-168

(d)

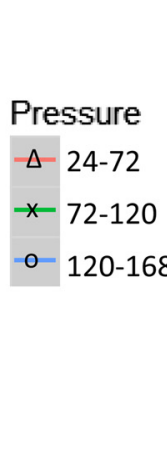

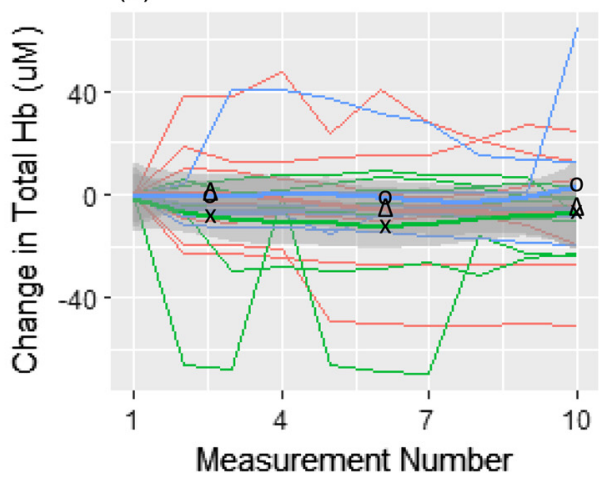

Pressure

$\triangle 24-72$

$72-120$

$120-168$

Fig. 7 For volunteer subject measurements, the total $\mathrm{Hb}$ measurement as a function of repeated measurement number is shown for each of the nine subjects at each of the three pressure levels. The moving average mean (loess) is also shown along with the $95 \%$ confidence intervals. This is shown for (a) cheek, (b) gum, (c) tongue, and (d) under the tongue. It can be seen that the total Hb largely does not show consistent or significant changes as a function of time for the tested conditions.

Finally, because a series of 10 repeated measurements were acquired from each site with the probe held in contact, it is possible to evaluate the dynamic changes in each endpoint as a function of time after the probe achieved the desired pressure level. The $\mathrm{Hb}$ saturation is shown as a function of measurement number (Fig. 6) for each of the four sites [(a) cheek, (b) gum, (c) tongue, and (d) under tongue]. The local mean and 95\% confidence intervals (CI) are plotted using the geom_smooth function from the ggplot2 package in $\mathrm{R}$ (indicating significant deviations from zero where the CI does not overlap the abscissa). ${ }^{15}$ Notably, the hemoglobin saturation shows a significant decrease over time for the highest pressure range for all tissue sites $(p<0.05$ for nonoverlapping $\mathrm{CI})$. There is some overlap in the CI among the different pressure levels, but the highest and lowest pressure levels are significantly separated for all sites except for the cheek at the later measurement times, with the higher pressure resulting in a larger reduction in hemoglobin saturation relative to the starting point. In contrast, total hemoglobin content does not appear to show consistent significant trends (Fig. 7).

\section{Discussion}

This article presents the development and validation of a pressure sensing, self-calibrated DRS system. Implementation of the automated pressure-sensing element with suitable control software addresses two important challenges in the implementation of a DRS system for physiologic assessment or diagnosis, (1) the influence of uncontrolled pressure on the physiologic status of the tissues being interrogated and (2) the temporal dependence of these physiologic parameters following initiation of probe contact. It was demonstrated that increasing applied pressure resulted in an increasing deviation, particularly for the case of $\mathrm{Hb}$ saturation, indicating that perfusion had been impeded and tissue oxygenation was reduced. This type of perturbation could influence diagnostic or prognostic systems in a clinical system, in particular for cancer diagnosis and therapy, where hypoxia is an important feature of tumor pathophysiology and a determinant of therapeutic response. It is worth noting that the expected change in blood volume with pulsatile flow is not likely to be significant. For example, Seo et al. ${ }^{17}$ found that in the visible range the RMS of the signal attributable to pulsatile changes was $<1 \%$ of the total blood volume in human skin, thus blanching of the tissue due to outflow of blood would be the expected major source of deviation with applied pressure. Physiologic changes in tumor oxygenation are driven by a variety of factors, including instability in red blood cell flux, vasomotion, and vascular reformation, with dominant changes occurring over the time frame of several cycles per hour or longer, making this an unlikely source of variability. ${ }^{18}$

The automated triggering of a measurement at a set pressure is also a critical feature developed here, given the short time scale over which the physiologic endpoints are changing (Figs. 6 and 7). This points to the need to acquire a measurement as soon as possible after the probe achieves sufficient pressure to ensure contact. In general, the lowest pressure settings showed smaller deviations, as compared to the higher pressures, as 
would be expected. To accurately sample patient tumors, which have a high degree of heterogeneity, multiple sampling points would ideally be taken both spatially and temporally, so this type of automated system would be critical.

In a diagnostic setting, the ability to reproducibly acquire data is critical to ensuring accuracy and reliability. This manuscript has demonstrated the significant deviations in physiologic parameters that can occur when using uncontrolled or relatively high pressure level measurements of tissue. For this reason, it is critical for contact measurements to integrate both a pressure sensor and automatic triggered measurement to ensure that any physiologic perturbation has been minimized. In this case, utilizing the $24 \mathrm{mmHg}$ setting with measurement acquired automatically as rapidly as possible would best preserve native tissue physiology.

\section{Disclosures}

Palmer, von Windheim, Ramunujam, Stevenson, Krieger, Koester, and Duke University have financial interest in Zenalux Biomedical, Inc., which produced and is commercializing the Zenascope instrument used.

\section{Acknowledgments}

The authors would like to acknowledge Yulin Zhao, who assisted with some of the animal measurements, as well as Amy Walker and Stephanie Martina who consented the volunteers. Funding was provided by the NIH (R42CA156901, JVW). No VA resources were used for this research. The views expressed in this article are those of the author (WTL) and do not necessarily represent the views of the Department of Veterans Affairs or the United States government.

\section{References}

1. B. Yu et al., "A self-calibrating fiber optic probe for tissue optical spectroscopy," in Conf. on Lasers and Electro-Optics (CLEO), San Jose, California (2008)

2. V. Chang et al., "Towards a field-compatible optical Spectroscopic device for cervical cancer screening in resource-limited settings: effects of calibration and pressure," Opt. Express 19(19), 17908-17924 (2011).

3. M. G. Nichols, E. L. Hull, and T. H. Foster, "Design and testing of a white-light, steady-state diffuse reflectance spectrometer for determination of optical properties of highly scattering systems," Appl. Opt. 36(1), 93-104 (1997).

4. N. M. Marin et al., "Calibration standards for multicenter clinical trials of fluorescence spectroscopy for in vivo diagnosis," J. Biomed. Opt. 11(1), 014010 (2006).

5. E. K. Chan et al., "Effects of compression on soft tissue optical properties," IEEE J. Sel. Top. Quantum Electron. 2(4), 943-950 (1996).

6. R. Reif et al., "Analysis of changes in reflectance measurements on biological tissues subjected to different probe pressures," J. Biomed. Opt. 13(1), 010502 (2008).

7. Y. Ti and W. C. Lin, "Effects of probe contact pressure on in vivo optical spectroscopy," Opt. Express 16(6), 4250-4262 (2008).

8. K. Vishwanath et al., "Quantitative optical spectroscopy can identify long-term local tumor control in irradiated murine head and neck xenografts," J. Biomed. Opt. 14(5), 054051 (2009).

9. F. Hu et al., "Rapid determination of oxygen saturation and vascularity for cancer detection," PLoS One 8(12), e82977 (2013).

10. B. Yu et al., "Diffuse reflectance spectroscopy with a self-calibrating fiber optic probe," Opt. Lett. 33(16), 1783-1785 (2008).

11. G. M. Palmer and N. Ramanujam, "Monte Carlo-based inverse model for calculating tissue optical properties. Part I: theory and validation on synthetic phantoms," Appl. Opt. 45(5), 1062-1071 (2006).

12. R. L. van Veen, W. Verkruysse, and H. J. Sterenborg, "Diffuse-reflectance spectroscopy from 500 to $1060 \mathrm{~nm}$ by correction for inhomogeneously distributed absorbers," Opt. Lett. 27(4), 246-248 (2002).
13. R. Huang and S. Jacques, "Skin optics summary," 1998, http://omlc.ogi. edu/news/jan98/skinoptics.html.

14. E. Jones et al., "SciPy: open source scientific tools for Python," 2001, http://www.scipy.org/ (08 May 2018).

15. R Core Team, R: A Language and Environment for Statistical Computing, R Foundation for Statistical Computing, Vienna, Austria (2016).

16. C. D. Willey et al., "Patient-derived xenografts as a model system for radiation research," Semin. Radiat. Oncol. 25(4), 273-280 (2015).

17. I. Seo, P. R. Bargo, and N. Kollias, "Simultaneous assessment of pulsating and total blood in inflammatory skin lesions using functional diffuse reflectance spectroscopy in the visible range," J. Biomed. Opt. 15(6), 060507 (2010).

18. M. W. Dewhirst, Y. Cao, and B. Moeller, "Cycling hypoxia and free radicals regulate angiogenesis and radiotherapy response," Nat. Rev. Cancer 8(6), 425-437 (2008).

Gregory M. Palmer is an associate professor of radiation oncology at Duke University, and directs the Duke Optical Molecular Imaging and Analysis shared resource, the Duke Small Animal Irradiator Facility, and the Duke Preclinical Translational Research Unit. He also works with Zenalux Biomedical Inc., which is commercializing technology developed in part during his $\mathrm{PhD}$ dissertation under Dr. Nirmala Ramanujam. His research interests include application of optical imaging and spectroscopy to study tumor physiology and treatment response.

Husam Mikati as an aspiring academic physician, is gearing work and academics to better understand the biology of cancer and how best to treat it. In the coming years, he will be receiving a master's in biomedical sciences and master's of biomedical and health informatics before going to medical school. Research and medicine have been his passions for years and he wants to continue to make contributions to both fields.

Joseph A. Herbert received his bachelor's degree in physics from the University of Mary Washington and his $\mathrm{PhD}$ in biomedical engineering from Virginia Commonwealth University. Currently, he is a postdoctoral researcher and project manager in Duke University's Preclinical Translational Research Unit. His work centers on increasing the translatability of preclinical research to successful clinical trials. His current research projects primarily involve developing novel radiation and chemotherapy treatments in tumor bearing mouse models.

Jesko von Windheim is a Duke professor of the practice and a technology entrepreneur who is focused on early-stage innovations in the physical sciences. He was a cofounder of Cronos Integrated Microsystems (acquired by JDS Uniphase) and CEO and founder of Nextreme Thermal Solutions (acquired by Laird Technologies). As a professor of the Practice, he works with faculty and students to identify, fund and commercialize early stage technologies for medical, environmental and industrial applications.

Nirmala Ramanujam is a Duke professor of biomedical engineering and global health. Her lab develops and applies innovative optical strategies and technologies for cancer screening in resource limited communities, detecting residual disease during surgeries, and visualizing tumor hypoxia and metabolism. She is also the founder of the Duke Global Women's Health Technologies Center.

Mark W. Dewhirst is the Gustavo S. Montana professor of radiation oncology. He has research interests in tumor hypoxia, angiogenesis, and drug transport. His work has focused primarily on translation of concepts in the laboratory to clinical testing. In the laboratory, he has spent over 30 years studying hypoxia and hyperthermia in tumors and has pioneered window chamber imaging techniques. Currently, he is a vice director for basic science in the Duke Cancer Institute and is active in faculty mentoring.

Walter T. Lee is an associate professor of surgery and codirector of the Head and Neck Program, Duke Cancer Institute Duke University Medical Center. He also serves as a staff surgeon in the Division of Otolaryngology-Head and Neck Surgery at the Durham VA Medical Center.

Biographies for the other authors are not available. 